\title{
Low-grade fibromyxoid sarcoma of the external anal sphincter: a case report
}

\author{
Lee-Kiat Ban ${ }^{1,2}$, Ailun Heather Tseng ${ }^{1}$, Shih-Hung Huang ${ }^{3 \dagger}$ and Henry Hsin-Chung Lee ${ }^{1,4,5^{*}+}$
}

\begin{abstract}
Background: Low-grade fibromyxoid sarcoma (LGFMS) is a rare soft tissue tumor that has a tendency to grow in the deep soft tissue of the trunk and extremities. Despite its benign appearance, the tumor has a high recurrence rate and metastatic potential. LGFMS in the perineal space is rare, and only a few cases have been reported. We present the first case of LGFMS to be located at the external anal sphincter.

Case presentation: A 27-year-old male patient admitted to our Surgical Department with perianal pain and swollen for a year. The digital rectal examination revealed a perianal mass. Oral metronidazole and analgesia were prescribed on suspicion of perianal abscess failed to alleviate the symptom; hence, the patient was scheduled for surgery. Intraoperative diagnosis revealed an encapsulated tumor in the external anal sphincter that extended from the perianal region orally to the pararectal space. The results of immunohistochemistry (MUC4 staining) and FUS gene rearrangement by fluorescence in situ hybridization confirmed the diagnosis of LGFMS.
\end{abstract}

Conclusions: This case is unique in terms of the location of the rare soft tissue tumor. Although LGFMS is considered low grade, its unpredictable behavior necessitates a long-term follow-up.

Keywords: Low-grade fibromyxoid sarcoma, LGFMS, External anal sphincter, Perineum

\section{Background}

Low-grade fibromyxoid sarcoma (LGFMS), a rare soft tissue tumor, was first described by Evans in 1987 [1]. Despite its deceptively benign appearance, LGFMS has a high tendency for local recurrence and late distant metastasis, mainly to the lung $[2,3]$. The histological appearance of LGFMS is characterized by the presence of alternating fibrous and myxoid area in a whorled growth pattern [3]. Although LGFMS is common in middle-aged adults, 13-19\% of cases occur in 18 years and younger. To date, the youngest reported case was 22-month-old $[2,4,5]$.

Given its bland appearance, LGFMS can be difficult to distinguish from some benign mesenchymal tumors and other low-grade sarcomas [3]. Epithelial membrane

*Correspondence: hhc.lee@gmail.com

${ }^{\dagger}$ Equal contributors

'Department of Surgery, Hsinchu Cathay General Hospital, Jhonghua Rd, Hsinchu, Taiwan

${ }^{4}$ Graduate Institute of Translational and Interdisciplinary Medicine, College of Health Sciences and Technology, National Central University, Jung-Da Rd, Taoyuan, Taiwan

Full list of author information is available at the end of the article antigen (EMA) is one of the diagnostic markers for LGFMS, but the poor specificity can also be detected in soft tissue perineurioma and a subset of solitary fibrous tumors [6-8]. Recently, Doyle et al. found that mucin 4 (MUC4) was a highly specific and sensitive marker for LGFMS when compared with other histologically similar tumors [3]. Furthermore, FUS gene rearrangement by fluorescence in situ hybridization (FISH) and FUS-CREB3L2/FUS-CREB3L1 chimeric fusion genes by reverse transcription polymerase chain reaction are the other two reliable approaches for LGFMS diagnosis [9].

We present the first case of a 27-year-old male with LGFMS in the external anal sphincter. The diagnosis of LGFMS was confirmed by MUC4 staining and FUS gene rearrangement using immunohistochemistry (IHC) and FISH, respectively.

\section{Case presentation}

A 27-year-old male with no relevant medical or surgical history who presented to the Surgical Department complaining of intermittent perianal pain for a year. He described a normal bowel habit of two to three times a day 
but rectal bleeding following defecation for 1 week. Physical examinations were unremarkable except a considerable redness, swelling, and tenderness on palpation over the perianal region. Digital rectal examination revealed a perianal tender mass. A prescription of oral metronidazole and analgesia for 1 week due to suspicion of perianal abscess failed to alleviate the symptom; hence, the patient was scheduled for surgical intervention. Intraoperative diagnosis revealed an encapsulated tumor in the external anal sphincter that extended from the perianal region orally to the pararectal space and there were no other abnormalities. The tumor was enucleated for definitive diagnosis.

The gross specimen, measuring $6.1 \times 4.6 \times 4.3 \mathrm{~cm}$, was tan and firm in appearance (Fig. 1). IHC analysis of the specimen showed an alternating myxoid and densely collagenous area with low-grade spindle cells (Fig. 2a-c). MUC4 stain (Fig. 2d) and FUS gene rearrangement by FISH (Fig. 3) were subsequently ordered for suspicion of LGFMS and all came back positive. The findings confirmed the diagnosis of LGFMS.

The patient underwent regular clinical follow-ups at 3-month interval and CT scan annually. One year after surgery, the patient was asymptomatic and in good health. The external anal sphincter muscle was fully functional, and the CT scan revealed no abnormal findings.

\section{Discussion}

The anatomic locations of LGFMS are common in the soft tissues of the upper limbs, lower limbs, and trunk wall $[2,5]$.

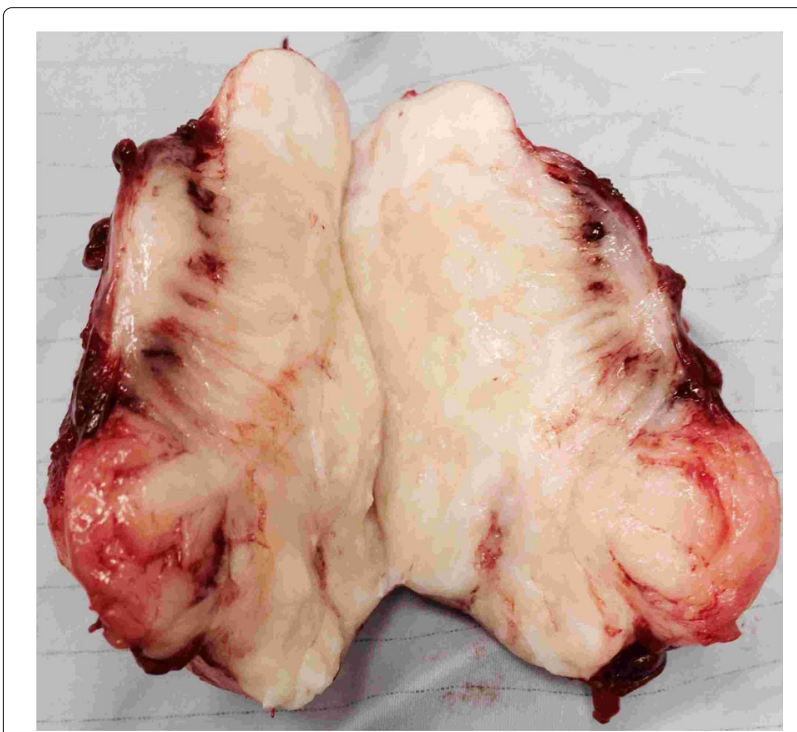

Fig. 1 Gross morphology of LGFMS from the 27-year-old patient. The resected tumor from the external anal sphincter is tan, firm, and has a glistening appearance
LGFMS occurring in the perineal space is extremely rare and only a few cases have been reported [10-17]. Park et al. published the first LGFMS in the colon of a 43-year-old man whose tumor was surgically removed along with the creation of right hemicolectomy combined with nephrectomy. The patient recovered well 24 months after surgery [17]. In another case, Mendoza and colleagues recently reported a 48-year-old female with LGFMS that arose from the sigmoid colon without metastatic disease. Despite the patient later developed a bowel obstruction that required another surgery, she ultimately recovered and was discharged to home; however, the follow-up information is not available [10]. Although LGFMS has a relatively good prognosis, the rate of local recurrence and late metastasis are high [15]. In 2011, Evans demonstrated an extensive follow-up study of 33 LGFMS cases with metastases. Of the 33 cases examined, 15 of them metastasized mostly to the lungs, pleura and chest wall, and occasionally to the pericardium. Other sites were also noted (two bones, one liver, and one heart). The interval to distant metastases varied up to 45 years with a median of 5 years, suggesting the indolent nature of the tumor [12].

The dilemma of LGFMS diagnosis lies on its benign appearance. IHC is a useful tool for diagnosis, but the markers are of limited values as the staining profile of LGFMS is nonspecific. For instance, we examined several markers such as desmin, S-100, CD34, and EMA, but the results were negative (data not shown). The findings are consistent with the published literatures $[2,10,15]$. Doyle and colleagues identified MUC4 as a highly sensitive and specific marker for LGFMS through gene expression profiling. To confirm the finding, 49 LGFMS cases were evaluated and all exhibited strong and diffuse cytoplasmic staining pattern [3]. However, when applied to suspicious MUC4-negative cases of LGFMS, FUS gene rearrangement should be exploited to guide the diagnosis $[9,18]$.

Surgical resection remains the standard treatment for LGFMS, with wide en bloc surgical resection being the most effective $[5,19]$ and enucleation, which was performed, being optimal for eliminating functional impairment of the anal sphincter muscle. We performed no adjuvant treatments because such practices to LGFMS are not conventional. Radiotherapy has effectiveness that remains in question to date and is performed only when factors such as margin positivity, tumor location, and tumor size indicate that recurrence or metastasis is likely. Chemotherapy is reserved for patients whose tumors recur locally or spread to distant sites [5, 20-22]. Overall, optimal treatment plans for maximizing oncologic control and delivering least functional impairments often involve multidisciplinary discussions. 
(A)

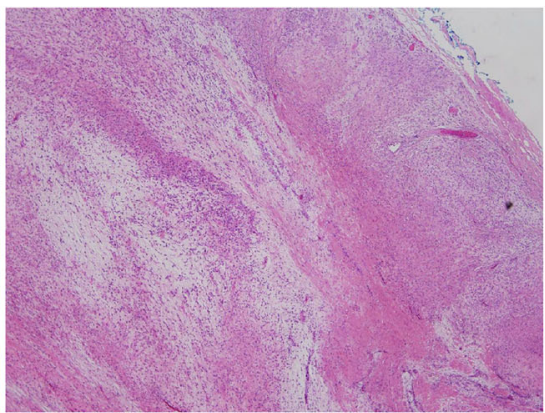

(C)

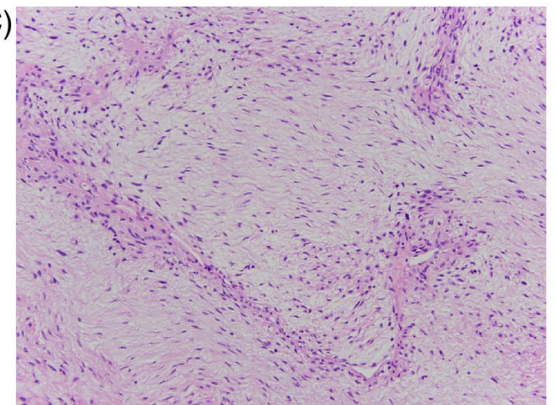

(B)

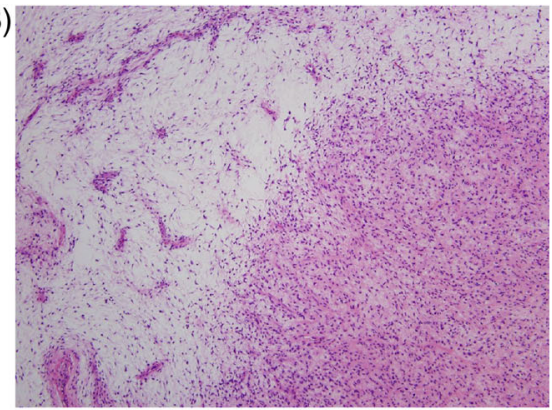

(D)

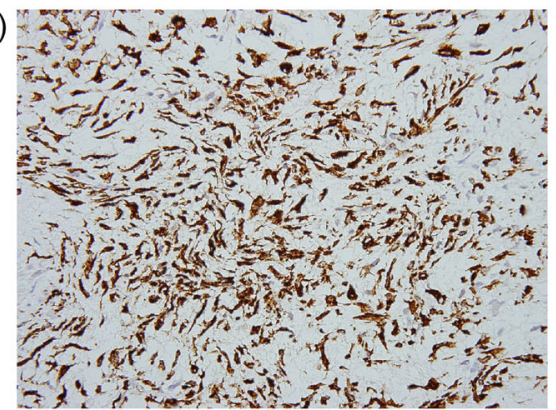

Fig. 2 Classic histological features of the LGFMS. Histological images of the LGFMS show $\mathbf{a}$ an admixture of collagenized and hypocellular myxoid zones (H\&E, $\times 40)$, b alternating hypocellular myxoid areas of whorled growth patterns (H\&E, $\times 100$ ), $\mathbf{c}$ bland-appearing small uniform spindle cells with arcades of small vessels (H\&E, $\times 200)$, and $\mathbf{d}$ strong immunoreactivity of MUC4 ( $\times 400)$

\section{Conclusions}

This case report enriches the literature with information on the site of LGFMS. Although the site of occurrence and benign appearance of LGFMS often make diagnosis difficult, the advancement in molecular pathology has proved

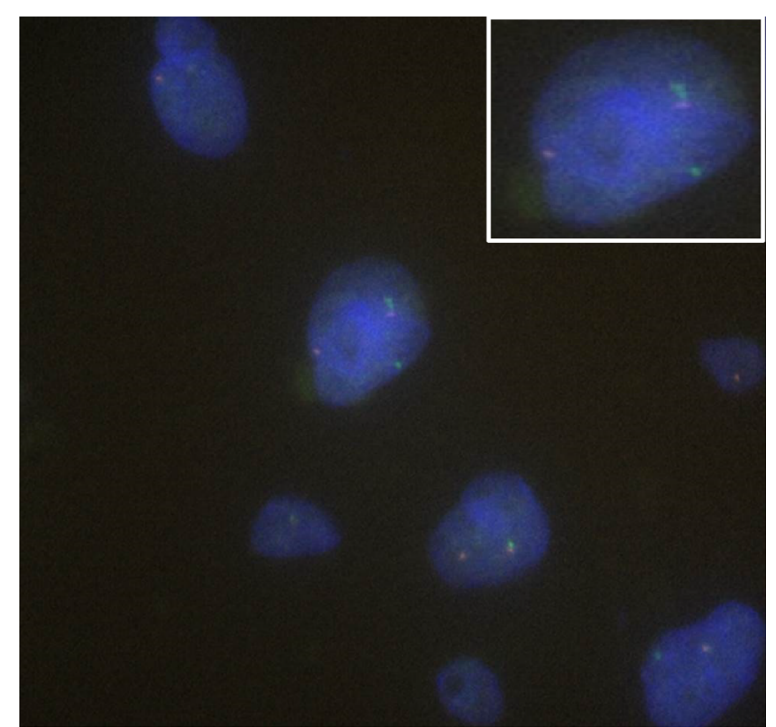

Fig. 3 Coexistence of fused- and split-signals of the FUS gene. FISH analysis of FUS (16p11) using dual-color probes detected both fusedand split-signals to be useful in clinical practices. Finally, the rate of occurrence and metastasis are high, and long-term follow-up is prudent.

\section{Abbreviations}

EMA: Epithelial membrane antigen; FISH: Fluorescence in situ hybridization; IHC: Immunohistochemistry; LGFMS: Low-grade fibromyxoid sarcoma; MUC4: Mucin 4

\section{Acknowledgements}

Not applicable.

\section{Funding}

No funding was provided.

\section{Availability of data and materials}

As a case report, all data generated or analyzed are included in this article.

\section{Authors' contributions}

AHT drafted this manuscript. LKB and HHCL analyzed and interpreted the patient data. SHH evaluated the histopathological images and prepared the figures. All authors read and approved the final manuscript.

\section{Competing interests}

The authors declare that they have no competing interests.

\section{Consent for publication}

Written informed consent for publication of the clinical details and/or clinical images was obtained from the patient. A copy of the consent form is available for review by the editor of this journal.

\section{Ethics approval and consent to participate}

The Ethics Committee of Cathay General Hospital, Hsinchu, Taiwan, approved this study. The analysis was performed in accordance with the ethical standards of the hospital. The patient reported in this study provided written consent. 


\section{Publisher's Note}

Springer Nature remains neutral with regard to jurisdictional claims in published maps and institutional affiliations.

\section{Author details}

${ }^{1}$ Department of Surgery, Hsinchu Cathay General Hospital, Jhonghua Rd, Hsinchu, Taiwan. ${ }^{2}$ College of Medical Technology, Nursing and Wellbeing, Yuanpei University of Medical Technology, Yuanpei Street, Hsinchu, Taiwan. ${ }^{3}$ Department of Pathology, Cathay General Hospital, Jen-Ai Road, Taipei, Taiwan. ${ }^{4}$ Graduate Institute of Translational and Interdisciplinary Medicine, College of Health Sciences and Technology, National Central University, Jung-Da Rd, Taoyuan, Taiwan. ${ }^{5}$ School of Medicine, Fu Jen Catholic University, Zhongzheng Rd, New Taipei, Taiwan.

Received: 9 February 2017 Accepted: 17 May 2017

Published online: 30 May 2017

\section{References}

1. Evans HL. Low-grade fibromyxoid sarcoma: a report of two metastasizing neoplasms having a deceptively benign appearance. Am J Clin Pathol. 1987;88(5):615-9.

2. Kurisaki-Arakawa A, Suehara Y, Arakawa A, Takagi T, Takahashi M, Mitani K, Kaneko K, Yao T, Saito T. Deeply located low-grade fibromyxoid sarcoma with fus-creb312 gene fusion in a 5-year-old boy with review of literature. Diagn Pathol. 2014;9(1):1.

3. Doyle LA, Möller E, Dal Cin P, Fletcher CD, Mertens F, Hornick JL. Muc4 is a highly sensitive and specific marker for low-grade fibromyxoid sarcoma. Am J Surg Pathol. 2011;35(5):733-41.

4. Folpe $A L$, Lane $K L$, Paull G, Weiss SW. Low-grade fibromyxoid sarcoma and hyalinizing spindle cell tumor with giant rosettes: a clinicopathologic study of 73 cases supporting their identity and assessing the impact of high-grade areas. Am J Surg Pathol. 2000;24(10):1353-60.

5. Tang Z, Zhou Z-H, Lv C-T, Qin LY, Wang Y, Tian G, Luo X-L, Zhu Q, Xu X-G. Low-grade fibromyxoid sarcoma: clinical study and case report. J Oral Maxillofac Surg. 2010;68(4):873-84.

6. Hornick JL, Fletcher CD. Soft tissue perineurioma: clinicopathologic analysis of 81 cases including those with atypical histologic features. Am J Surg Pathol. 2005;29(7):845-58.

7. Mentzel T, Schärer L, Kazakov DV, Michal M. Myxoid dermatofibrosarcoma protuberans: clinicopathologic immunohistochemical, and molecular analysis of eight cases. Am J Dermatopathol. 2007;29(5):443-8.

8. Pelmus M, Guillou L, Hostein I, Sierankowski G, Lussan C, Coindre JM. Monophasic fibrous and poorly differentiated synovial sarcoma: immunohistochemical reassessment of $60 \mathrm{t}(\mathrm{x} ; 18)$ (syt-ssx)-positive cases. Am J Surg Pathol. 2002;26(11):1434-40.

9. Patel RM, Downs-Kelly E, Dandekar MN, Fanburg-Smith JC, Billings SD, Tubbs RR, Goldblum JR. Fus (16p11) gene rearrangement as detected by fluorescence in-situ hybridization in cutaneous low-grade fibromyxoid sarcoma: a potential diagnostic tool. Am J Dermatopathol. 2011;33(2): 140-3.

10. Mendoza AS, O'Leary MP, Peng SK, Petrie BA, Li Al, French SW. Low-grade fibromyxoid sarcoma of the sigmoid colon. Exp Mol Pathol. 2015;98(2):300-3.

11. Alevizopoulos A, Mygdalis V, Tyritzis S, Stravodimos K, Constantinides CA. Low-grade fibromyxoid sarcoma of the renal pelvis: first report. Case Rep Nephrol Dial. 2012;2(2):87-91.

12. Evans HL. Low-grade fibromyxoid sarcoma: a clinicopathologic study of 33 cases with long-term follow-up. Am J Surg Pathol. 2011;35(10):1450-62.

13. Harish K, Ashok A, Alva N. Low grade fibromyxoid sarcoma of the falciform ligament: a case report. BMC Surg. 2003;3(1):1.

14. Koishi A, Gomibuchi H, Inoue J, Minoura S, Itoh E, Saito M. Hyalinizing spindle cell tumor with giant rosettes of the omentum. J Obstet Gynaecol Res. 2003;29(6):388-91.

15. Konecna J, Liberale G, Haddad J, de Saint-Aubain N, El Nakadi I. Diffuse intra-abdominal low grade fibromyxoid sarcoma with hepatic metastases: case report and review of the literature. Int J Surg Case Rep. 2015;14:40-3.

16. Laurini JA, Zhang L, Goldblum JR, Montgomery E, Folpe AL. Low-grade fibromyxoid sarcoma of the small intestine: report of 4 cases with molecular cytogenetic confirmation. Am J Surg Pathol. 2011;35(7):1069-73.
17. Park I, Kim H, Yu C, Kim J, Jang S, Kim J. Low-grade fibromyxoid sarcoma of the colon. Dig Liver Dis. 2007;39(3):274-7.

18. Lin G, Doyle LA. An update on the application of newly described immunohistochemical markers in soft tissue pathology. Arch Pathol Lab Med. 2015;139(1):106-21.

19. Gonzalez LJ, Artigas RV, Rodríguez BM, Lopez-Pousa A, Bagué S, Abellan M, Trias FM. Differences between en bloc resection and enucleation of retroperitoneal sarcomas. Cirugia Espanola. 2014;92(8):525-31.

20. Maretty-Nielsen K, Baerentzen S, Keller J, Dyrop HB, Safwat A. Low-grade fibromyxoid sarcoma: incidence, treatment strategy of metastases, and clinical significance of the FUS gene. Sarcoma. 2013;2013:256-80.

21. Ramu EM, Houdek MT, Isaac CE, Dickie Cl, Ferguson PC, Wunder JS. Management of soft-tissue sarcomas; treatment strategies, staging, and outcomes. SICOT J. 2017;3:20.

22. Zagars GK, Ballo MT, Pisters PW, Pollock RE, Patel SR, Benjamin RS, Evans HL. Prognostic factors for patients with localized soft-tissue sarcoma treated with conservation surgery and radiation therapy. Cancer. 2003;97(10):2530-43.

\section{Submit your next manuscript to BioMed Central and we will help you at every step:}

- We accept pre-submission inquiries

- Our selector tool helps you to find the most relevant journal

- We provide round the clock customer support

- Convenient online submission

- Thorough peer review

- Inclusion in PubMed and all major indexing services

- Maximum visibility for your research

Submit your manuscript at www.biomedcentral.com/submit
C Biomed Central 